\title{
Self and body esteem perception in multiple sclerosis ${ }^{1}$
}

\author{
Yoram Barak ${ }^{\mathrm{a}, *}$, Yair Lampl ${ }^{\mathrm{b}}$, \\ Ida Sarova-Pinchas ${ }^{\mathrm{b}}$ and Anat Achiron ${ }^{\mathrm{c}}$ \\ ${ }^{a}$ Yehuda Abarbanel Mental Health Center, Bat Yam, \\ Israel \\ ${ }^{\mathrm{b}}$ Department of Neurology, Edith Wolfson Medical \\ Center, Holon, Israel \\ ${ }^{\mathrm{c}}$ M.S. Center, Sheba Medical Center, Tel-Hashomer, \\ Israel
}

Self esteem and body esteem were examined in a group of 35 relapsing-remitting multiple sclerosis (MS) patients using the Body Esteem Scale (BES) and the Eysenck Self Esteem Scale (ESES) and compared to age and sex matched normal controls.

There were 23 females and 12 males in the MS patient's group; average age 38.9 years (range: 22-52). All participants completed the self-rated BES evaluating the following subscales: females - sexual attractiveness, physical condition and weight concern; males - physical attractiveness, physical condition and upper body strength. In addition all participants were scored, following a semi-structured interview, on the ESES. Psychiatric co-morbidity was excluded using a semi-structured interview by the consulting psychiatrist. All evaluations were carried out during the remitting phase. Statistical analysis, comparing patients to healthy controls, demonstrated lowered self-rating of the physical condition (males $<0.05$, females $<0.001)$. On the other hand, no significant differences were found in the physical (male) or sexual (female)attractiveness subscales. The mean ESES score in the patients group was $23.2 \pm 4.0$, slightly above the reported average. The controls mean ESES was $28.4 \pm 3.6,(P<0.05)$. No correlation was found between self and body esteem amongst M.S. patients. This study emphasizes impaired perception of body esteem in multiple sclerosis patients even in remission. The preservation of physical and sexual attractiveness may be related to the non-disfiguring nature of the disease. Preservation of self-esteem in MS patients suggests that body-esteem should be the focus of supportive treatment.

\section{Introduction}

Body image is a term that is often used in the psychological and psychiatric literature [14]. It has even been implicated in the etiology of certain psychiatric

\footnotetext{
${ }^{1}$ The institutes where the authors are working are all affiliated with the Sackler Faculty of Medicine, Tel Aviv University.

* Corresponding author: Yoram Barak, Abarbanel Mental Health Center, 15 Keren-Kayemet Street, Bat-Yam 59110, Israel. Fax: +972 3 5552738; E-mail: mdybarak@netvision.net.il.
}

disorders [1]. Nevertheless, exact definition and instruments (subjective and objective) for the evaluation of the perception of self esteem and its important component - body image - have been lacking in previous years. The self scored Body Esteem Scale (BES) has been developed and validated by Franzoi and Shields [3] and offered a quick reliable measurement of this perception.

Assessment of Body-esteem should be supplemented by evaluation of self-esteem, as it is important to unravel the extent to which these two concept are interwined. In the 'Ego and the Id' Freud stated that 'the ego is first and foremost a bodily ego' [4]. Thus the human body's shape and movement reflect inner physical and psychological states intimately related to self-esteem. One of the commonly used models to estimate (quantitatively) emotional stability is based on the fact that various traits are empirically found to correlate together, in the formation of such stability. The main traits are self-esteem, anxiety, autonomy and guilt feelings [2]. People who score high on self-esteem tend to have confidence in themselves and their abilities.

Multiple sclerosis (MS) patients report various sexual difficulties and dysfunctions [11,13]. Although often related to the disease process itself, psychopathology affecting these patients may be a contributing factor. Co-morbidity of depression [7, 9] or neurosis [10] in MS patients can adversely influence sexuality and feelings of attractiveness. This study focuses on the perceptions of self and body esteem in MS patients in order to evaluate these important psychological dimensions, and their possible implications to the patients coping with their disease.

\section{Subjects and methods}

\subsection{Subjects}

Twenty-three females and 12 males with relapsingremitting MS (definite - according to Poser's criteria [8]), were evaluated. Patients were recruited from a random sample attending the Sheba Medical Center 
MS clinic during a one month period. Average age of the patients group was 38.9 years (range: 22-52). All patients were in the remitting phase of the disease. The mean Kurtzke [5] EDSS scores in the group were 4.1 (S.D. \pm 1.4 ). Inclusion criteria were: (a) definite diagnosis, (b) Kurtzke score less than 6.0 (ambulatory) and (c) informed consent. Exclusion criteria were: (a) severe obesity (over $140 \%$ of ideal body weight), (b) disfiguring features (i.e., scars, burns, angioma, etc.) and (c) present or past history of psychiatric disorder.

The control group consisted of 41 age and sex matched healthy controls. Average age was 36.4 years (range: 23-59). These were volunteers recruited amongst students at a night school. Exclusion criteria were identical to those of the MS group.

\subsection{Methods}

The study was approved by both the local and the Ministry of Health ethical committees.

Each patient was examined, independently, by two neurologists to confirm the diagnosis and rate the Kurtzke EDSS score. Psychiatric evaluation was carried out by a psychiatrist based on a semi-structured interview. Following this initial evaluation, all patients completed the self-rated Body Esteem Scale (BES). The BES is a self-rated questionnaire evaluating different aspects of the body esteem [3]. The subscales differ for males and females as follows: (a) females: ' $\mathrm{S}$ ' - sexual attractiveness, 'P' - physical condition and ' $\mathrm{W}$ ' - weight concern; (b) males: 'A' - physical attractiveness, 'P' - physical condition and ' $U$ ' - upper body strength (representing virility).

The ESES was completed by the interviewing psychiatrist, after interviewing patients and controls. The questionnaire consists of 30 questions, each scored as 1 point for the correct answer. The average reported by Eysenck for 12000 tested subjects is 21.5 [2].

Statistical analysis was performed using the Unpaired Student $t$ Test and the Spearman Rank Order correlations.

\section{Results}

Thirty-five MS patients and 41 controls completed the interview and both questionnaires.

The MS patients' BES scores were as follows: (a) females: ' $\mathrm{S}$ ' - median 48.3 (range: 39-51); ' $\mathrm{P}$ ' - median 29.4 (range: 23-35); 'W' - median 41.5 (range: 35-49) and (b) males: ' $A$ ' - mean 42.0 (range:
35-51); 'P' - median 40.7 (range: 32-48); 'U' - median 22.5 (range: 18-27).

The control subjects' BES scores were as follows: (a) females: ' $\mathrm{S}$ ' - median 50.5 (range: 43-57); ' $\mathrm{P}$ ' - median 36.2 (range: 28-42); 'W' - median 37.2 (range: 28-42) and (b) males: 'A' - median 42.7 (range: 34-51); 'P' - median 47.6 (range: 31-53); 'U' - median 26.0 (range: 22-31).

Both male and female MS patients showed a significant decrease in esteem of their physical condition (' $\mathrm{P}$ ' males $p<0.05$; 'P' females $p<0.001$ ). The males; upper body strength subscale and the females' weight concern subscale were marginally different between the patients and the controls $(0.02<$ 'P' $<0.05)$.

The subscales relating to self esteem of physical and sexual attractiveness were found not to be significantly different between controls and patients $(p=0.84-$ males; $p=0.87$ - females) No statistical correlation was found between the BES score, as well as its subscales for males and females, and the Kurtzke scale $(p=0.5)$.

The patients mean ESES score was $23.4 \pm 4.0$, compared with a score of $28.4 \pm 3.6$ for controls $(P<0.05)$. The Spearman correlation test showed a coefficient of $R^{2}=0.43$ between self and body esteem, not statistically Significant $(P=0.112)$.

\section{Discussion}

One half to two thirds of MS patients described having changes in their sex lives after the beginning of the symptoms [13] and $15 \%$ of those patients already have symptoms of sexual problems at the time of diagnosis [11]. The different authors are not in agreement whether the sexual disturbances are only due to the central nervous system involvement or to additional psychological mechanisms of coping.

In the present study we evaluated MS patients perception of self and body esteem in order to further clarify the possible psychological impairment and its relation to self esteem, physical and sexual attractiveness.

The instruments used in this study were a multidimensional questionnaire [3] rating different aspects of body esteem, and a rater scores questionnaire quantifying self-esteem. Patients of both sexes showed a significant decrease in esteem of their physical condition. This decrease is probably related to the physical disability present in MS patients. Although in our series all patients were ambulatory (Kurtzke EDSS 
less than 6.0), in the remitting phase, disability was not absent and was probably reflected in the decreased physical condition subscale.

In contrast, the male patients' esteem of physical attractiveness and the female patients' perception of sexual attractiveness were not significantly reduced. These findings contradicted the hypothesis that MS patients refuse to engage in sexual activity if they perceive themselves as unattractive and repulsive [13]. There are several explanations that may clarify this surprising finding. MS is not a disfiguring disease, so that external appearance is not damaged. Psychological compensation through mechanisms of denial or reaction formation may be playing a part in this population as an aid to coping with the disease. Further supporting this assumption are the results of the ESES. First, although lower than normal subjects scores, patients' scores were within limits considered to reflect normal self-esteem. Second, we think that disability was the major factor contributing to lowered bodyesteem. Previous studies demonstrated that correlations between measures of body satisfaction and selfesteem are negligible in disabled subjects [6], where as in the non disabled the correlations are substantial. Our results confirm these findings showing that in MS patients there is a tendency to devaluate body-esteem in the composite self-esteem.

The present study demonstrates conserved perception self esteem, of physical and sexual attractiveness in MS patients. The sexual dysfunctions reported in MS patients are probably related to organic impairment, and the contribution of psychological factors, (such as lowered self-esteem), studied is less pronounced, These results can be applied in constructing psychotherapeutic interventions. Focus of therapy should be shifted to physical rehabilitation and support.

\section{References}

[1] H. Bruch, Perceptual and conceptual disturbances in anorexia nervosa, Psychosomatic Med. 24 (1962), 187-194.

[2] H.J. Eysenck and G. Wilson, Know your own personality, Maurice Temple Smith, London, 1975, pp. 67-91.

[3] S.L. Franzoi and S.A. Shields, The body esteem scale: multidimensional structure and sex differences in a college population, J. Person. Assess 48(2) (1984), 173-178.

[4] S. Freud, The ego and the id, Haggard, London, 1923, pp. 1953-1966.

[5] J.F. Kurtzke, Rating neurologic impairment in multiple sclerosis: an expanded disability status scale (EDSS), Neurology 33 (1983), 1444-1452.

[6] J.D. Mayer and M.G. Eisenberg, Self-concept and the spinalcord-injured: an investigation using the Tennessee Selfconcept Scale, J. Consult. clin. Psychol. 50 (1982), 604-605.

[7] G.P. McIvor, M. Rilikan and M. Reznikoff, Depression of multiple sclerosis as a function of length and severity of illness, age, remission and perceived social support, J. Clin. Psychol. 40 (1984), 1028-1053.

[8] C.M. Poser, D.W. Paty and L. Scheinber, New diagnosis criteria for multiple sclerosis: guidelines for research protocols, Ann. Neurol. 13 (1983), 227-231.

[9] R.B. Schiffer, The spectrum of depression in multiple sclerosis, Arch. Neurol. 44 (1987), 596-599.

[10] K. Skegg, P.A. Corwin and B.C.G. Skegg, How often is multiple sclerosis mistaken for a psychiatric disorder?, Psychol. Med. 18 (1988), 733-736.

[11] E. Stenager, L. Knudsen and K. Jensen, When should the patient with multiple sclerosis be told his diagnosis?, in: Mental Disorders and Cognitive deficits in Multiple Sclerosis, $\mathrm{K}$. Jensen, L. KnudsenI. Grant, eds, John Libbey, London, 1989, pp. 191-195.

[12] E. Stenager, E.N. Stenager and J.K. Jensen, Sexual aspects of multiple sclerosis, Seminars in Neurology 12(2) (1992), $120-124$.

[13] J. Vas, Sexual impotence and some autonomic disturbances in men with multiple sclerosis, Acta Neurol. Scand. 45 (1969), 166-182.

[14] C.D. van der Velde, Body image of one's self and others: Developmental and clinical significance, Am. J. Psychiatry 142 (1985), 527-537. 


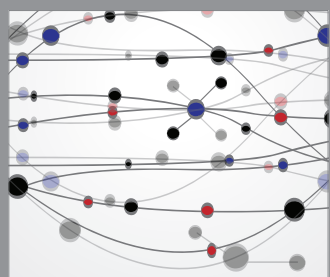

The Scientific World Journal
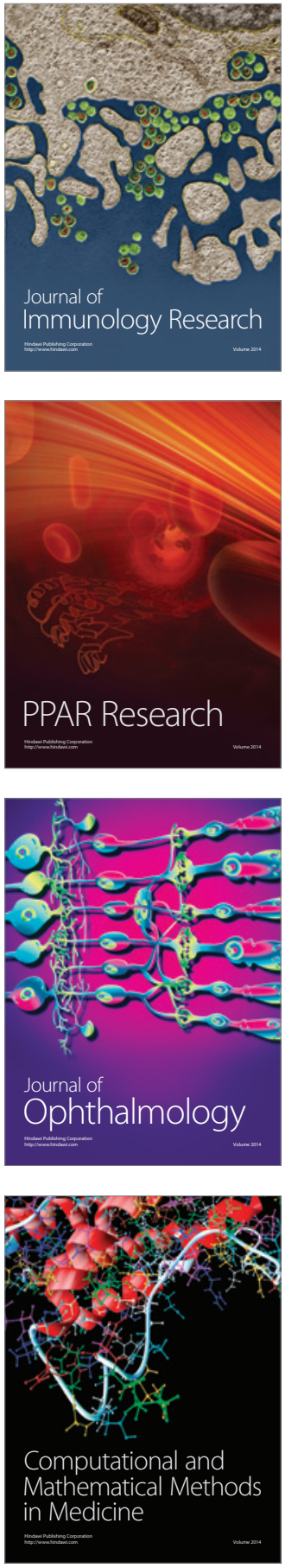

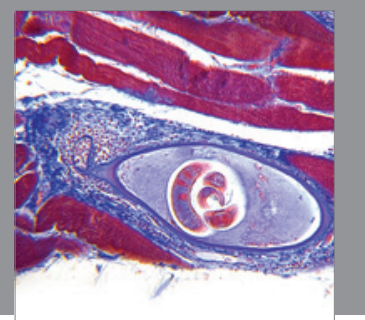

Gastroenterology

Research and Practice
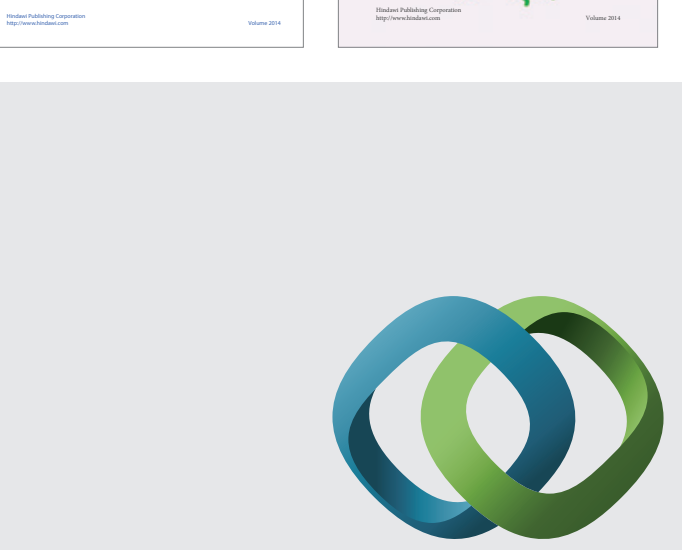

\section{Hindawi}

Submit your manuscripts at

http://www.hindawi.com
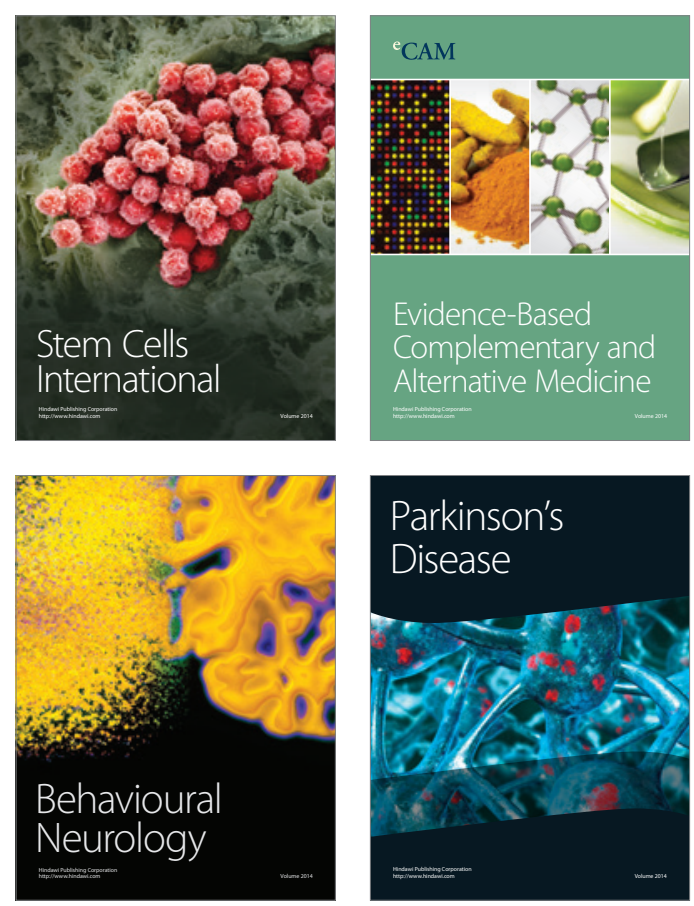

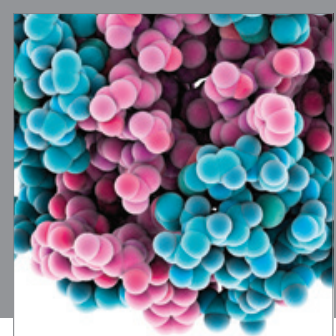

Journal of
Diabetes Research

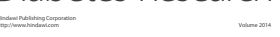

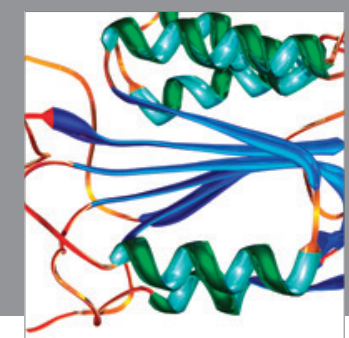

Disease Markers
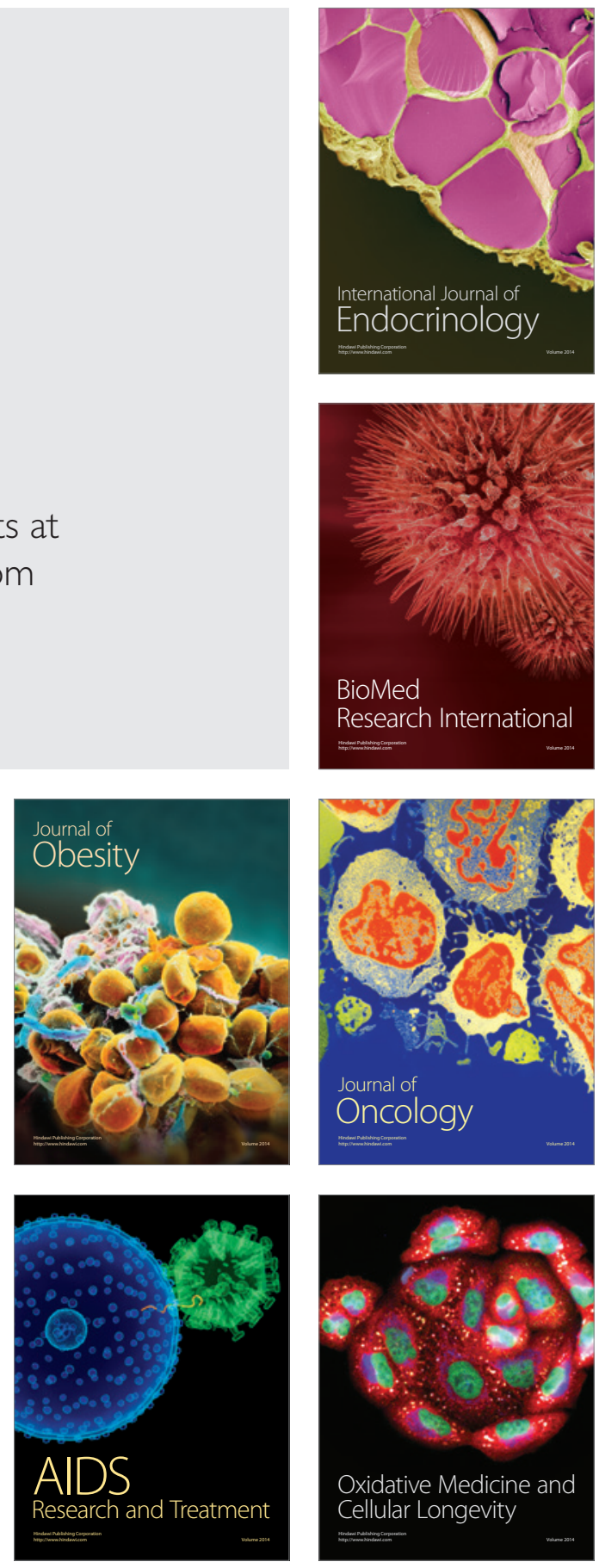